\title{
CONTRIBUTIONS TO GENETIC ALGEBRAS II
}

\author{
by HARRY GONSHOR
}

(Received 11th July 1972)

\section{Introduction}

This paper is a continuation of (4). The main aim of this paper is the introduction of the concept of sex-linked duplication. In addition, we shall give several equivalent definitions for the concept of a genetic algebra and make several remarks on overlapping of generations.

\section{Sex-linked duplication}

In analogy with duplication, one can define the concept of sex-linked duplication. However, caution is required since a natural naïve approach which first comes to mind fails. Consider the simple gametic algebra with basis $D$ and $R$, where $D^{2}=D, R^{2}=R$, and $D R=\frac{1}{2} D+\frac{1}{2} R$. . With basis $a=D$ and $b=D-R$ we obtain $a^{2}=a, a b=\frac{1}{2} b$, and $b^{2}=0$. The sexlinked algebra ((2), Section 4) has a basis $D \times D, D \times R, R \times R, D, R$, with, for example, $(D \times R) D=\frac{1}{4}(D \times D)+\frac{1}{4}(R \times D)+\frac{1}{4} D+\frac{1}{4} R$ (we are improving the notation used in (2)). By calculation

$$
(D \times D)(D-R)=\frac{1}{2}(D \times D-D \times R)=\frac{1}{2}[D \times(D-R)]=\frac{1}{2}(a \times b) .
$$

The naïve approach, which involves pretending as if $a$ and $b$ are genotypes and following the usual rules for sex linkage, would give

$$
(D \times D)(D-R)=(a \times a) b=\frac{1}{2}[a a \times b+a a]=\frac{1}{2}[a \times b+a] .
$$

Hence, unlike the case of duplication, it is not in general correct to apply the expected rule with a basis other than the original.

Nevertheless, a theory of sex-linked duplication is possible. We consider a baric algebra $A$ with weight $w$, i.e. we include the weight into the structure we are considering. The sex-linked duplication algebra of the algebra $(A, w)$ is the algebra $C$ with underlying vector space $A \times A \oplus A$ where $A \times A$ is as in (4, Section 3), and product defined as follows:

$$
(a \times b \oplus c)(d \times e \oplus f)=\frac{1}{2}(a b \times f \oplus w(f) a b)+\frac{1}{2}[d e \times c \oplus w(c) d e] .
$$

If we identify $a \times b$ with $a \times b \oplus 0$ and $c$ with $0 \oplus c$ we obtain the following:

$$
\begin{aligned}
(a \times b)(c \times d) & =0 \text { for all } a, b, c, d \in A \\
a b & =0 \text { for all } a, b \in A \\
(a \times b) c & =c(a \times b)=\frac{1}{2}[a b \times c+w(c) a b] .
\end{aligned}
$$


We first show that the operation is well defined. Define

$$
(a, b) \cdot c=\frac{1}{2}[a b \times c+w(c) a b]
$$

and extend by linearity to a multiplication of elements in $G$ by elements of $A$. Since - is trilinear in $a, b$, and $c$ and symmetric in $a$ and $b$, the product of an element of $I$ and an element of $A$ is 0 . Hence this induces a multiplication of elements of $A \times A$ by elements of $A:(a \times b) \cdot c=\frac{1}{2}[a b \times c+w(c) a b]$. Thus we obtain an algebra with the table as given above. This agrees with the definitions in the special cases discussed in (2).

Now $x \in C$ can be expressed uniquely as $x_{1}+x_{2}$ where $x_{1} \in A \times A$ and $x_{2} \in A$.

$C$ is not baric. In fact, $C$ has a basis consisting of nilpotent elements. However, we shall see that $C^{2}$ is baric. For $x=a \times b+c$ define $w^{\prime}(x)=w(c)$ and $w^{\prime \prime}(x)=w(a) w(b)$. It is easy to see that $w^{\prime}$ and $w^{\prime \prime}$ extend to linear functions on C. (For $w^{\prime \prime}$, we extend as usual to a bilinear function on $G$ and note that we obtain 0 if either factor is in $I$.) Now if $\mathrm{x}=(a \times b) \cdot c$, then

and

$$
w^{\prime \prime}\left(x_{1}\right)=\frac{1}{2} w^{\prime \prime}(a b \times c)=\frac{1}{2} w(a b) w(c)=\frac{1}{2} w(a) w(b) w(c)
$$

$$
w^{\prime}(x)=w\left(x_{2}\right)=\frac{1}{2} w(c) w(a b)=\frac{1}{2} w(a) w(b) w(c) .
$$

Hence by linearity, for arbitrary $c \in C^{2}, w^{\prime}(c)=w^{\prime \prime}(c)$. Note also that

$$
w^{\prime}[(a \times b) \cdot c]=\frac{1}{2} w^{\prime \prime}(a \times b) w(c) .
$$

Using bilinearity, if $x \in A \times A$ and $y \in A$ then $w^{\prime}(x \cdot y)=\frac{1}{2} w^{\prime \prime}(x) w(y)$. Let $D$ be the subset of $C$ consisting of all elements $x$ such that $w^{\prime}(x)=w^{\prime \prime}(x)$. We have shown that $C^{2}$ is included in $D$. It follows that $D$ is a subalgebra of $C$. We now show that $D$, and hence $C^{2}$, is baric. Finally, let $x, y \in D$. Then

$$
\begin{gathered}
w^{\prime}(x y)=w^{\prime}\left[\left(x_{1}+x_{2}\right)\right. \\
\left.\left(y_{1}+y_{2}\right)\right]=w^{\prime}\left(x_{2} y_{1}+x_{1} y_{2}\right)=w^{\prime}\left(x_{2} y_{1}\right)+w^{\prime}\left(x_{1} y_{2}\right) \\
=\frac{1}{2} w\left(x_{2}\right) w^{\prime \prime}\left(y_{1}\right)+\frac{1}{2} w^{\prime \prime}\left(x_{1}\right) w\left(y_{2}\right)=\frac{1}{2} w^{\prime}(x) w^{\prime}(y)+\frac{1}{2} w^{\prime}(x) w^{\prime}(y) \\
=w^{\prime}(x) w^{\prime}(y) .
\end{gathered}
$$

Since it is clear that $w^{\prime}$ is non-trivial on $C^{2}$, hence on $D, w^{\prime}$ is a weight. Hence $D$ is baric.

We shall show later that $D$ is a genetic algebra. The proof will be given by means of a convenient basis-free definition of a genetic algebra.

As in the base of duplication, the property of being special train is not preserved. Use the ordinary zygotic algebra with basis $a, b, c$ and table $a^{2}=a$, $a b=\frac{1}{2} b, b^{2}=\frac{1}{4} c, c a=c b=c^{2}=0 . \quad I=(a \times b, b \times b, a \times c, b \times c, c \times c, b, c)$. Now $\quad b \times b=\frac{1}{4}(a \times b)(b) \in I^{2}$. However, $(a \times a+a)(b \times b)=\frac{1}{8}(a \times c+c) \notin I^{2}$ since $I^{2}=(a \times b, b \times b, a \times c, b \times c, c \times c)$.

We note that if $a b=0$ in $A$ then $(a \times b) x=0$ for all $x$ in $C$.

Sections 4 and 5 of (2) can be redone in the spirit of this section to make them more transparent. We shall not pursue this exercise here. 
Sex-linked duplication is not as applicable as duplication. For example, if the formalism is attempted in the case of polyploidy the genetic significance becomes obscure. Nevertheless, the operation does lead to the correct result in many genetic systems.

\section{Definitions for a genetic algebra}

In this section we shall introduce certain equivalent definitions for the concept of a genetic algebra. Although some of the equivalences appear to be known, they are not, as far as we know, found in the literature.

We let $A$ be a baric algebra and $N$ the ideal of elements with weight 0 . The right powers $N^{r}$ of the ideal $N$ where $r$ is an integer are defined inductively as $N^{1}=N$ and $N^{n+1}=N^{n} N$. Note that for non-associative algebras $N^{r}$ is not necessarily an ideal and $N^{r} N^{s}$ does not necessarily equal $N^{r+s}$. We also define two other types of powers. $N^{[n]}$ is the set of all linear combinations of products of elements of $A$ with at least $n$ terms from $N . N^{(n)}$ is the set of all linear combinations of right products of elements of $A$ with at least $n$ terms from $N$. Clearly $N^{(n)}$ and $N^{[n]}$ are ideals.

We summarise the basic properties of these powers.

\section{Lemma}

(i) $N^{(n+1)}$ is the smallest ideal containing $N^{(n)} N$;

(ii) $N^{(r)}=N^{(r+1)} \Rightarrow N^{(r)}=N^{(s)}$ if $s>r$;

(iii) $N^{[r]} N^{[s]} \subset N^{[r+s]}$;

(iv) $N^{(r)} \subset N^{[r]}$;

(v) $N^{[2 r-1]} \subset N^{(r)}$.

The elementary proof is left to the reader. Consider the special train algebra with basis $\left\{a_{0}, a_{1}, a_{2}, a_{3}\right\}$ and multiplication table:

$$
a_{i} a_{j}=a_{i+j} \text { unless } i=j=2 \text { and } a_{2} a_{2}=a_{3} \text {. }
$$

(We are using the usual convention that $a_{i+j}=0$ if $i+j>3$.)

In this case

$$
\begin{aligned}
& N=\left(a_{1}, a_{2}, a_{3}\right), \quad N^{(2)}=\left(a_{2}, a_{3}\right), \quad N^{(3)}=\left(a_{3}\right), \quad N^{(4)}=0, \\
& N^{[2]}=\left(a_{2}, a_{3}\right), \quad N^{[3]}=\left(a_{3}\right), \quad N^{[4]}=\left(a_{3}\right), \quad N^{[5]}=0 .
\end{aligned}
$$

It follows from the table that this is a uniform counterexample which shows that the first three parts of the Lemma are false if ( ) and [ ] are interchanged and that the inclusion in (iv) cannot be reversed. More generally, consider the algebra with basis $\left\{a_{0}, a_{1}, \ldots, a_{n}\right\}$ with multiplication table:

$$
a_{i} a_{j}=a_{i+j} \text { unless } i=j \text { and } a_{i} a_{i}=a_{i+1} .
$$


This example shows that the result in (v) is sharp in the sense that $N^{\left[2^{r-1}\right]} \subset N^{(r+1)}$ is not always valid.

We are now ready to state the following theorem:

Theorem 3.1. Let $A$ be a baric algebra and $N$ the ideal of elements with weight 0. Then the following conditions are equivalent:

(1) $A$ is a genetic algebra;

(2) $N^{(n)}=0$ for some $n$;

(3) $N^{[n]}=0$ from some $n$;

(4) If $B$ denotes multiplication by $b$ in the multiplication algebra $M(A)$, then $b \in N \Rightarrow B \in \operatorname{rad} M(A)$.

Proof. It is clear that $(1) \Rightarrow(2)$. In fact, if $a_{0}, a_{1}, \ldots, a_{n}$ is a basis of $A$ with table satisfying:

$$
\begin{aligned}
a_{i} a_{j}=\Sigma \lambda_{i j k} a_{k} \text { where } \lambda_{000} & =1 \\
\text { for } k<j & \lambda_{0 j k}=0
\end{aligned}
$$

and for $i, j>0$ and $k \leqq \max (i, j), \lambda_{i j k}=0$

then $N=\left(a_{1}, a_{2}, \ldots, a_{n}\right)$ and $N^{(n+1)}=0$. (2) $\Leftrightarrow$ (3) follows from the inclusions

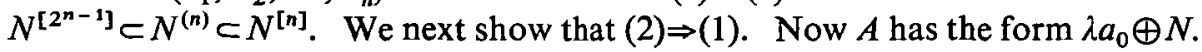
Let $N=N^{(1)} \supset N^{(2)} \supset \ldots \supset N^{(r)}=0$. Let $1 \leqq s \leqq r-1$. Since $N^{(s)}$ and $N^{(s+1)}$ are ideals, $A_{0}$ operates in a natural way on the vector space $N^{(s)} / N^{(s+1)}$. Furthermore for $b \in N, B$ operates on $N^{(s)} / N^{(s+1)}$, in fact $B N^{(s)} / N^{(s+1)}=0$. Hence there exists a basis of $N^{(s)} / N^{(s+1)}$ with respect to which $A_{0}$ is lower triangular. $B$ is, of course, 0 regardless of the basis! If the bases for the various $s$ are lifted in any manner, we obtain a basis for $N$. It is now easily seen that with respect to $a_{0}$ and the new basis for $N$ the algebra is genetic. (The argument is the same as that of the last part of the proof of Theorem 2.1 in (4).)

To prove that (2) $\Rightarrow(4)$ suppose $b \in N$ and $N^{(n)}=0$. Consider the right ideal $B M(A)$. Since $A[B M(A)]^{n} \subset N^{(n)}=0$. Thus $B \in B M(A) \subset \operatorname{rad} M(A)$. Conversely, $B \in \operatorname{rad} M(A)$ for all $b \in N$ implies that $A N^{(r)}=0$ for some $r$. It follows that $N^{(r+1)}=0$. This completes the proof.

Remarks. The above use of quotient spaces could also be used to simplify the proof of Theorem 2.1 in (4). Incidentally, as an alternative source to the text on Lie algebras mentioned in (4), we mention (6) and the references there.

Definitions (2) or (3) may be used to give an alternative proof that $A \times A$ is genetic if $A$ is genetic. Note first that $M$, the ideal of elements of weight 0 in $A \times A$ is $N \times A$. It is easy to see that $M^{(2)} \subset N^{(2)} \times A+N \times N$. More generally, we have the following table of inclusions for $r \geqq 1$ where by convention we let $N^{0}=N^{(0)}=N^{[0]}=A$ :

$$
\begin{aligned}
& M^{r} \subset N^{r-1} \times N ; \\
& M^{(r)} \subset N^{(r)} \times A+N^{(r-1)} \times N ;
\end{aligned}
$$




$$
M^{[r]} \subset \sum_{s=0}^{r}\left(N^{[s]} \times N^{[r-s]}\right) .
$$

(4) and (5) are easily proved by ordinary induction. (6) is easily seen by complete induction. The result that $A$ genetic $\Rightarrow A \times A$ genetic is clear from the table.

The following Theorem can now easily be proved.

Theorem 3.2. If $A$ is a genetic algebra with weight $w$ and $C$ is the sex-linked duplication algebra, then the subalgebra $D$ of $C$ consisting of all elements $x$ such that $w^{\prime}(x)=w^{\prime \prime}(x)$ is a genetic algebra.

In this case $M$, the ideal of elements of weight $0=N \times A+N$. We obtain the following table for $r \geqq 2$ :

$$
\begin{aligned}
& M^{r} \subset N^{r-1} N \times ; \\
& M^{(r)} \subset N^{(r-1)} \times N+N^{(r)} \times A+N^{(r)} ; \\
& M^{[r]} \subset \sum_{s=0}^{r}\left(N^{[s]} \times N^{[r-s]}\right)+N^{[r]} .
\end{aligned}
$$

In the special case where $N A=N$ the above inclusions become equalities. Note first that the above condition implies that $A^{2}=A$ since $N=N A \subset A^{2}$ and $A^{2}$ necessarily contains an element of weight different from zero. The verification is then routine. Note that although it is easy to construct genetic algebras with $N A \neq N$, (such as e.g. the mutation algebra in (2, Section 3) where $r+s=1$ ), most algebras studied in (2), (3), and (4) satisfy $N A=N$.

It is interesting to note that in this case $M^{(r)}$ and $M^{[r]}$ are ideals even in the original algebra $A \times A+A$.

We make two final remarks concerning the types of powers studied here. First, a special train algebra may be defined as a genetic algebra satisfying $N^{r}=N^{(r)}$.

It is clear from the tables that duplication and sex-linked duplication rarely lead to special train algebras. For example, if $N A=N$ and $N^{2} \neq 0$ it is clear that in either case $M^{2} \neq M^{(2)}$.

Secondly, the polyploidy algebras studied in (3) satisfy $N A=N$ as well as $N^{(r)}=N^{[r]}$. Furthermore, in the special case studied in $(2), N^{(r)} / N^{(r+1)}$ is 1-dimensional for all $r$ such that $N^{(r)} \neq 0$. It might be worth while to give special names to algebras having such desirable properties; however, we shall not pursue this subject here.

\section{Overlapping of generations}

We consider a generalisation of the situation discussed in (1, Section 3$)$. The usual implicit assumption that mating occurs at discrete intervals is kept, but we assume that a fixed ratio of a population in a given generation survive into the next generation. We also assume that the survivors are indistinguishable from the new generation with respect to further breeding. Certainly a more 
refined study in population genetics would require distinguishing between a $D_{i}$ survivor and a new $D_{i}$ as well as a consideration of selection and assortative mating. If the original table corresponding to the case without overlapping had the form $D_{i} D_{j}=\sum_{k} \lambda_{i j k} D_{k}$ then the new table has the form

$$
D_{i} D_{j}=r \sum_{k} \lambda_{i j k} D_{k}+(1-r) \frac{1}{2}\left(D_{i}+D_{j}\right)
$$

for some fixed $r$. This may be regarded as a mixture of algebras as discussed in (4, Section 5) where the first is the original algebra and the second is a gametic multiple allelic algebra (see 3, Section 2). Now the latter algebra is genetic with respect to any basis where the first term represents a population and the other terms have the sum of their coefficients zero. Hence the mixture is a genetic algebra. Of secondary interest is the remark that if we start with a special train algebra, then the mixture is also a special train algebra.

\section{Miscellaneous remarks}

The original proof found for Theorem 3.2 was made with an explicit basis and was much more computational. It will suffice to indicate the basis and ordering here. The computation itself is more or less routine.

Suppose $A$ is genetic with respect to the basis $a_{0}, a_{1}, a_{2}, \ldots, a_{n}$. We know that $\left\{a_{i} \times a_{j}: i \leqq j\right\} \cup\left\{a_{i}\right\}$ is a basis for $C$.

In the future, for convenience we replace $a_{i} \times a_{j}$ by $a_{i j}$. Note that $w\left(a_{i j}\right)=0$ unless $i=j=0$ and $w\left(a_{00}\right)=1$. We thus can obtain a basis for $D$ by deleting $a_{00}$ and $a_{1}$ and inserting $a_{00}+a_{0}$. It is immediate that this gives us a subspace of $C$ of deficiency 1 all of whose elements satisfy $w\left(x_{1}\right)=w\left(x_{2}\right)$. Hence this subspace is identical to $D$.

Now $a_{0} a_{i}$ has the form $\lambda_{i} a_{i}+\sum_{k>i} \lambda_{0 i k} a_{k}$. Let $\mu_{i}$ be a root of the equation $x^{2}-\lambda_{i} x-\lambda_{i}=0$. Note that $\mu_{i}$ is real if $\lambda_{i}$ is real and non-negative. We choose a new basis for $D$ by replacing $a_{0 i}$ by $b_{i}=a_{0 i}-\mu_{i} a_{i}$ for all $i>0$. We use the convention: $\max \left(a_{i j}\right)=\max (i, j), \max a_{i}=\max b_{i}=i, \min \left(a_{i j}\right)=\min (i, j)$, $\min \left(b_{i}\right)=0, \min \left(a_{i}\right)=-1$ and $\max \left(a_{00}+a_{0}\right)=\min \left(a_{00}+a_{0}\right)=0$. Then we define the ordering on the basis as follows:

$x_{i} \geqq x_{j}$ iff $\max x_{i}>\max x_{j}$ or $\max x_{i}=\max x_{j}$ and $\min x_{i} \geqq \min x_{j}$.

(5) which has recently come to our attention introduces the concept of a dibaric algebra. It is easy to see that a sex-linked duplication algebra is dibaric. Section 3 of the latter paper introduces partial sex linkage. The corresponding multiplication table can be defined for a general baric algebra $A$ as follows:

Let the underlying vector space be $(A \otimes A) \oplus(A \times A)(A \otimes A$ is the usual tensor product and $A \times A$ is as in Section 2). Then define the product as:

$$
\begin{aligned}
(a \otimes b)(c \otimes d) & =(a \times b)(c \times d)=0 \\
(a \otimes b)(c \times d)=(c \times d)(a \otimes b) & =\frac{1}{2}(1-\theta)[w(b)(a \times c d)+w(a)(c d \otimes b)] \\
& +\frac{1}{2} \theta[w(a)(b \times c d)+w(b)(c d \otimes a)] .
\end{aligned}
$$


It is easy to see that this is well defined and consistent with the definition in (5). The algebra is clearly dibaric. In this case, $M$, the ideal of elements of weight 0 , is $N \otimes A+A \otimes N+N x A$. The following table is easy to obtain : For $r \geqq 2$

$M^{r} \subset N^{r-1} \times N+N^{r-1} \otimes N$;

$M^{(r)} \subset N^{(r-1)} \times N+N^{(r)} \times A+N^{(r-1)} \otimes N+N \otimes N^{(r-1)}+N^{(r)} \otimes A+A \otimes N^{(r)}$

$M^{[r]} \subset \sum_{s=0}^{r} N^{[s]} \times N^{[r-s]}+\sum_{s=0}^{r} N^{[s]} \otimes N^{[r-s]}$.

Again, it follows from the table that the algebra is genetic if $A$ is genetic.

\section{REFERENCES}

(1) ANDREOLI Givolio, Algebre non associative e sistemi differenziati di Riccati in un problema di Genetica, Ann. Mat. Pura Appl. (4) 49 (1960), 97-116.

(2) H. Gonshor, Special train algebras arising in genetics, Proc. Edinburgh Math. Soc. (2) 12 (1960), 41-53.

(3) H. Gonshor, Special train algebras arising in genetics II, Proc. Edinburgh Math. Soc. (2) 14 (1965), 333-338.

(4) H. Gonshor, Contributions to genetic algebras, Proc. Edinburgh Math. Soc. (2) 17 (1971), 289-297.

(5) P. Holgate, Genetic algebras associated with sex linkage, Proc. Edinburgh Math. Soc. (2) 17 (1970), 113-120.

(6) R. SHock, Nil subrings in finiteness conditions, Amer. Math. Monthly 78, (1971), 741-748.

Rutgers College

NEW BRUNSWICK

NEW JERSEY 08903 